\title{
Knowledge-Based System for Manufacturing Sustainability
}

\author{
Antonio Giovannini* ${ }^{* * *}$, Alexis Aubry ${ }^{*}$, Hervé Panetto ${ }^{*}$, Michele Dassisti ${ }^{* * *}$, Hind El Haouzi ${ }^{* * *}$ \\ * CRAN, Université de Lorraine, CNRS, Campus scientifique \\ B.P. 70239 F-54506 Vandouvre-lès-Nancy Cedex, France \\ (e-mail: \{antonio.giovannini; alexis.aubry;herve.panetto\}@univ-lorraine.fr). \\ ${ }^{* *}$ TRANE SAS, rue des Amériques, 88190 Golbey, France \\ (e-mail: hind.elhaouzi@trane.com). \\ *** DIMEG, Politecnico di Bari, Viale Japigia, 70100 Bari, Italy \\ (e-mail:m.dassisti@poliba.it).
}

\begin{abstract}
Today, sustainability becomes one of the biggest challenges. It represents a key issue in every production activity. To face this issue, a possible solution is to enhance knowledge usage in manufacturing and sustainability domains. In this paper, we extend the ONTO-PDM ontology for formalizing sustainable manufacturing knowledge. An industrial case is presented for instantiating the extension. Moreover we design a knowledge-based system, which exploits sustainable manufacturing knowledge for supporting design and process planning with sustainability proposals, generating machine code starting from product specifications.
\end{abstract}

Keywords: Sustainability, Manufacturing Processes, Knowledge-Based Systems, Ontology, Design, Process Planning

\section{INTRODUCTION}

Sustainability has been often considered as the revolution of the 21 st Century and today it represents a key challenge for each manufacturing enterprises. Sustainable manufacturing is defined as the creation of manufactured products that use processes that minimize negative environmental impacts, conserve energy and natural resources, are safe for employees, communities, and consumers and are economically sound (U.S. Department of Commerce, 2009).

In our view, the most effective way for infusing sustainable manufacturing knowledge is to support decision makers during their usual tasks. Our aim is to use sustainability concepts for elaborating design and process planning solutions, which allow reducing products sustainability cost.

To cope with this issue, in this paper, we extend ONTOPDM (Tursi et al., 2009; Panetto et al., 2012) with concepts and rules on sustainability principles and technology knowledge. Moreover, we design a Knowledge-Based System (KBS) that use formalized knowledge for supporting design and process planning. The use of ONTO-PDM in the system allows solving information interoperability issues between different product information sources. The resulting system is able to use formalized knowledge and product-related information in enterprises databases (DBs) for automatically generating machine code. Interfaces allow the system easily to adapt to different production sites and accepting experts interventions such as knowledge updating or business rules imposition.

Next sections are structured as follows: in section 2.1, we briefly describe the history of KBS, focusing on design and manufacturing planning support, and the advantage of ontologies usage in these systems; in section 2.2, related works on ontology for manufacturing and sustainability are presented; in section 3, the ONTO-PDM extension structure is described; in section 4 we show how the ontology could work in a real multi-site industrial scenario; in section 5 we propose the design of a KBS to exploit the ontology; section 6 is devoted to conclusions and future perspectives.

\section{BACKGROUND}

\subsection{From Manufacturing KBS to Ontology usage}

From 1976, with the first KBS (or expert system) (Freitas et al., 2005), the growth of this type of systems never stops. Already in 1988, authors in (Iwata, 1988) cope with the status of KBS, most of all in the manufacturing domain. Design and process planning support already dealt with a lot of domain issues (Tab.1).

Tab. 1 - Extract of a table on KBS applications (Iwata, 1988)

\begin{tabular}{|c|c|}
\hline $\begin{array}{l}\text { FIELD OF } \\
\text { APPLICATION }\end{array}$ & SUBJECT OF APPLICATION \\
\hline Design & $\begin{array}{l}\text { - Understanding of functional requirements } \\
\text { - Preparation and confirmation of specifications } \\
\text { - Recognition and understanding of specifications } \\
\text { - Examination and determination of basic concept of } \\
\text { design } \\
\text { - Formation of product concept } \\
\text { - Formation of model } \\
\text { - Formation od computational and experimental } \\
\text { procedures } \\
\text { - Analysis and simulation of structure } \\
\text { - Inspection of drawings } \\
\text { - Documentation of design }\end{array}$ \\
\hline $\begin{array}{l}\text { Manufacturing } \\
\text { Planning }\end{array}$ & $\begin{array}{l}\text { - Understanding of functional requirements for production } \\
\text { - Formation of manufacturing model } \\
\text { - Process planning } \\
\text { - Operation planning }\end{array}$ \\
\hline
\end{tabular}


A KBS consists of four main components (D. Pham et al., 1988): a knowledge base (KB) containing knowledge about the problem to solve; an inference engine to use the knowledge stored to obtain solutions to problems; a user interface for explaining reasons of resulting solutions; a knowledge acquisition module to develop/update the system (not always available).

The development of KBS in mid ' 80 is the main reason for the use of ontologies in computer science and artificial intelligence (AI) (Freitas et al., 2005). Ontology is an explicit specification of a conceptualization (Gruber, 1993). Through the use of ontologies for representing knowledge, this can be understood, shared and communicated across people and computers (Darai et al., 2010). The development of ontologies as a tool for knowledge representation has been a revolution in knowledge engineering, so that according to (Staab, 2009), AI researchers have highlighted the need of a more robust and theoretically sound engineering ("ontology engineering") which enables knowledge sharing/reuse and formulation of the problem solving process itself.

For a KBS, the knowledge base development is the most expensive task (Freitas et al., 2005). Hence, it is easy to understand the great opportunity given by ontologies. In fact, in early KBS, knowledge was about specific tasks while ontologies formalize knowledge about a domain improving expertise reusability. Therefore, ontologies are natural candidates as knowledge bases in KBS.

\subsection{Ontologies in manufacturing and sustainability}

In the manufacturing context, there have been some efforts in developing ontologies, at different levels, for supporting information exchange and reuse and creation of new knowledge. A consistent number of ontologies developed in this domain are mainly taxonomies (i.e., particular classifications arranged in a hierarchical structure and organized by generalization-specialization relationships or parent-child relationships). Really few research works reached the effective development of a formal ontology.

Some ontologies have been developed so far in the field of manufacturing. Lemaignan et al. (Lemaignan et al., 2006) propose an ontology applied to manufacturing, namely MASON (MAnufacturing's Semantics Ontology). MASON has been built upon three head concepts: entities, operations, and resources. Main applications of this ontology are: 1) to develop architectures and tools for automatic cost estimation; 2) to link a high level ontology with a multi-agent framework for manufacturing simulation.

To achieve an effective and efficient support to product design decisions, Wang and Tong (Wang et al., 2008) proposed an ontological approach to analyse what kinds of manufacturing knowledge are needed for achieving design decisions. An ontology in the domain of manufacturing knowledge has been thus designed by using the ontology development tool Protégé 3.3.1 with the intention to investigate its application in design decision support.
In (Tursi et al., 2009; Panetto et al., 2012), authors have developed a product ontology, named ONTO-PDM, based on two standards, for providing a semantic layer to business, design and manufacturing product-related information (in the section 3, a description of this work is presented with more details).

The knowledge management in industrial scenarios, instead of its modelling, has been the objective of the Know-Ont ontology of (Harshit Kumar et al., 2010). The proposed ontology is used to provide reusability of stored information in an efficient and effective way.

Quite a few authors have faced the problem of ontologies for sustainability. Many of the sustainability factors in manufacturing do interact, influence, restrict and depend on each other. This complexity is the main reason why an ontology may be a suitable tool to successfully reach the target of a sustainable manufacturing (Kibira et al., 2009).

Authors in (Reyes-Cordoba et al., 2008) stress the role of knowledge management coupled together with management actions to support decisions concerning the different engineering activities that have to be performed for waste minimisation. The authors adopt an ontology for mapping and organising the whole knowledge to provide an effective way for reducing gaps in the information needed to fully understand the process and identify the signs that point towards waste generation.

In (Brilhante et al., 2006) the authors present a very general framework based on an ontology and metadata, in connection with a software implementation, to analyse systems sustainability making use of quantitative and/or qualitative sustainability indicators.

In a preliminary study, (Dori et al., 2005) stress on shifting from an older energy-driven paradigm to a knowledgedriven one related to manufacturing. For building a comprehensive ontology, the authors firstly face the problem of knowledge mapping by adopting an Object Process Methodology-based approach for modelling product lifecycle knowledge.

In (Ceccaroni et al., 2004) a decision-support system for wastewater management is presented. The strength of the proposed application is the possibility to manage the complexity of multidisciplinary knowledge necessary for wastewater management in supporting the DSS, particularly for qualitative knowledge.

In (Garrido et al., 2011) the authors propose an ontology for impact assessment by including natural language definitions. The system supports the environmental indicator assessment contributing information about the state of the ecosystem or something relative to it. The same ontology is devised as a support to a decision support system for environment impact assessment.

In this section we have reviewed KBS solutions and ontology for sustainability and manufacturing. To our best of knowledge, no KBS for supporting design and process planning are able to support these tasks with sustainable manufacturing proposals. 


\section{A KBS FOR SUSTAINABLE MANUFACTURING}

In each enterprise, product-related information flow is managed by heterogeneous applications; users and domains that have to interoperate according to this shared heterogeneous information. This heterogeneity causes a "babel tower effect" that makes manufacturing sustainability principles harder to apply. To solve the information sources heterogeneity issue, (Tursi et al., 2009; Panetto et al., 2012) proposed a product-centred information view. Then, they developed the ONTO-PDM product ontology extending and formalising this concept.

In this paper, we utilize a part of the ONTO-PDM ontology of (Tursi et al., 2009; Panetto et al., 2012), translated in OWL (McGuinness et al., 2004), in order to assure information interoperability between applications that contain product-related data. ONTO-PDM was based on two standards, STEP - PDM and IEC 62264.

We extend the IEC part of ONTO-PDM for describing sustainable manufacturing concepts. Rules based on sustainable manufacturing principles need to be formalized for performing sustainability suggestions. The aim is then to use all product-related information for proposing better solutions from the sustainability point of view.

We formalize sustainability concepts essentially to perform proposition about resources type, resources quantity, resources way to use, processes and/or process parameters changes for improving the overall product sustainability.

To suggest changes that do not affect product functional features, we have added classes in IEC resources and process models. In the Material Model (Fig. 1) and Equipment Model we added classes (MaterialUse and EquipmentUse), which link an instance of Material/EquipmentClass with their functions (e.g. galvanized steel sheets $\rightarrow$ exposure to weather condition).

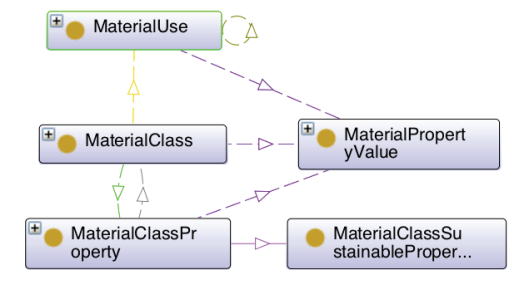

Fig. 1 - Modifications in the IEC Equipment Model.

Each use corresponds to a set of material/equipment properties values (MaterialPropertyValue). An appropriate SWRL rule (Horrocks et al., 2004) makes possible to infer a relationship between materials (rule below) or equipment and their function as follows:

MaterialClass(?a),MaterialUse(?b),MaterialPrope rtyValue(?c),MaterialPropertyValue(?d),Material ClassProperty(?e), hasPropertyValue(?a,?c), hasPr opertyValue(?b,?d), hasPropertyValue(?e,?c), hasP ropertyValue(?e,?d), maxValue(?d,?f),minValue(?d ,?g), maxValue(?c,?h), minValue(?c,?i), unitofMeas ure(?d,?l),unitofMeasure(?c,?m),lessThanOrEqual
$(? h, ? f)$, less ThanOrEqual(?g,?i), stringEqualIgnore Case(?l,?m)->materialUse(?a,?b),

A material is considered suitable for replacing the previous one if it has the same use and a property of the MaterialClassSustainableProperty (Fig. 1). This class is a subset of MaterialClassProperty. Appropriate rules guide inferences identifying instances, which can be adopted to propose sustainable production solutions. For instance, the SWRL rule

\section{MaterialClass(?a),MaterialClass(?b), MaterialUse (?c),MaterialClassSustainableProperty(?d),Materi alSpecification(?e),ProductSegment(?f), materialU se(?a,?c),materialUse(?b,?c), hasPropertyOf(?b,?d ), is ACollectionOf(?f, ?e),specifies(?e, ?a)- $>$ E_materialProposal $(? b, ? e)$}

allows to identify material changes opportunity.

To allow process proposals, we have added classes in the ProcessSegment Model (Fig. 2). A process segment represents a process with related resources required and process parameters. The class Process specifies the generic process, without resources requirements or parameters related. The class ProcessGoal identifies the reasons to use a process. The sustainability of a process or of a process segment is evaluated by means of a subset of process parameters (SustainableParameter). SustainableParameter class is a subclass of Parameter and has the same function of MaterialClassSustainableProperty for the Material Model. Also in this case, SWRL rules support the identification and proposition of processes that have the same goal of the previous selected ones,

Process(?a),Process(?b),ProcessGoal(?c),Sustain ableParameter(?d),ProductSegment(?f), ProcessSe gment(?g),process(?g,?a),goal(?a,?c),goal(?b,?c), hasParameter $(? b, ? d)$, correspondesTo(?f, ?g)$>$ processProposal(?f, ?b)

or process segments that have the same process of the previous selected ones,

\section{ProcessSegment(?a),ProcessSegment(?b),Process( ?c), SustainableParameter(?d),ProductSegment(?f) ,process $(? a, ? c), p r o c e s s(? b, ? c)$, hasParameter $(? b, ?$ d), correspondesTo(?f, \\ $>$ processSegmentProposal(?f, ?b) \\ $? a)-$}

but that are better from a sustainability point of view.

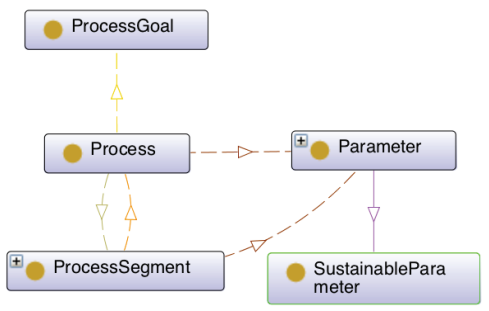

Fig. 2 - Modifications in the IEC ProcessSegment Model. 
If experts accept a suggestion, other resources, processes and /or product part features can be affected. In Fig. 3 the general structure of the ontology subpart, which describe relations between changes is presented. The SustChange class, in fact, is strongly related with resources, processes and/or product parts parameters involved in the product components manufacturing processes. Sustainable resources, processes and process parameters (EquipmentClass, MaterialClass, Process, Parameter) are related to the SustChange individuals that "manage" the changes, containing information about modifications on the use of other sets of resources (EquipmentUse, MaterialUse) and/or modification about set of processes to perform (ProcessGoal) and/or modification about how to perform them (Parameter).

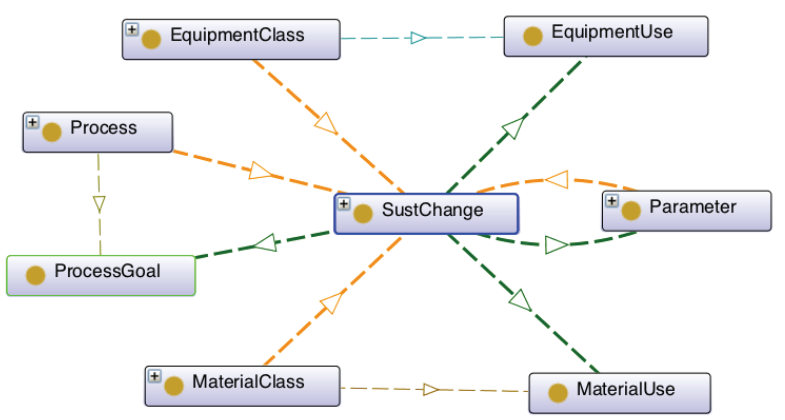

Fig. 3 - Extract of extended ontology: these OWL classes express knowledge about relations between sustainability proposals.

Appropriate SWRL rules use these relations for highlight changes caused by the acceptance of a suggestion. For instance the rule

MaterialClass(?c),MaterialClassSustainablePrope
rty(?d),MaterialSpecification(?b),ProductSegment
(?a),SustChange(? e),Process(?f),ProcessSegment(
?g),Parameter(?h),effect(?c,?e), hasPropertyOf(?c,
?d), isACollectionOf(?a,?b),specifies(?b,?c),chang
e(?e,?f),technology(?g,?f),correspondesTo(?a,?g),
change(?e,?h)->hasParameter(?g,?h)

proposes a process parameter change caused by the acceptance of a material with sustainable properties.

\section{INDUSTRIAL CASE}

In this section we exploit an industrial case to instantiate the extension of the ontology and to test the inferences based on the SWRL rules presented in the previous section. The case here presented concerns a multinational company (Trane) working in the sector of air conditioning and refrigeration units for industrial and commercial use. Currently Trane is dealing with the sustainability issue.

Trane products are characterized by modularity. Every module needs several sheet metal parts. Sheet metal parts are involved in a punching and a bending process. In Fig. 4 , is shown the example of ontology instantiation with information about product assembling configurations of sheet metal parts in a Trane product module. In Fig. 4, is possible to highlight the sheet metal parts of a sample module.

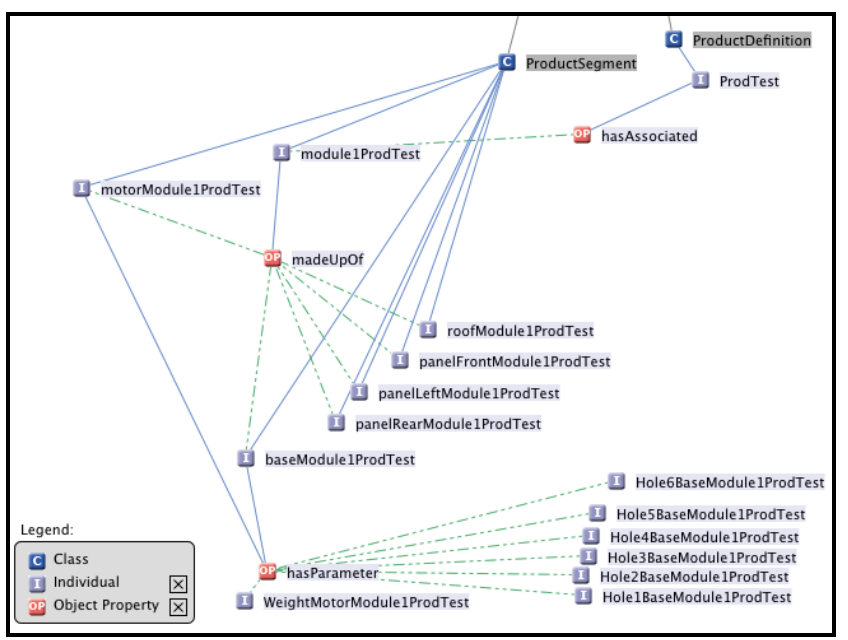

Fig. 4 - Extract of the Product Definition Model partially instantiated with a Trane product.

Currently most of products are for external use, therefore under weather conditions exposure. Galvanized steel is the main used material. When in the ontology, we introduce the corrosion performances of galvanized steel the inference engine, thanks to SWRL rules in previous section, is able to inference that this steel is for external use (Fig. 5). Moreover in the Fig. 5, it is shown that the $\mathrm{KB}$ can highlight the possibility to replace galvanized steel with aluminium that is more recyclable than the first one.

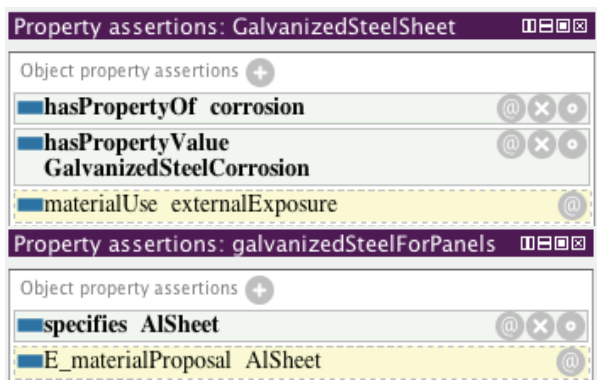

Fig. 5 - Inference results on material use and sustainable suggestion.

If, in Trane, a decision maker accepts the suggestion, the inference engine is able to propose changes on other equipment material and/or process involved in the product manufacturing. In Fig. 6, the inference is shown, based on the SWRL rules, of a parameter (bending force), and proposed after the aluminium choice.

\begin{tabular}{|c|c|}
\hline Property as sertions: bending 1 & 由日口回 \\
\hline Object property assertions + & \\
\hline process bendingMetal & $x$ \\
\hline
\end{tabular}

Fig. 6 - Inference results on process parameter.

\section{KB SYSTEM DESIGN PROPOSAL}

\subsection{Knowledge to be formalized}

To support experts in design and process planning, it is required knowledge related to product assembling 
configuration, technologies, resources and product design constraints.

Knowledge about product assembling configurations can be instantiated, in IEC 62264, into the Product Definition Model. Relationships between them, as well as parts design features, have to be formalized as rules. Thanks to the IEC Product Definition Model (Fig. 4), it is possible to structure information concerning components features, assembling and resources (material, equipment and personnel) required to manufacture a part. This information and rules like If I have to manufacture a module to contain a compressor of $X \mathrm{~kg} \rightarrow$ are required $Y$ holes on the module base sheet metal, can be used to recognize holes number and shape in one of the sheet metal parts, which support the module content (motors, filters, etc.). Moreover information about product modules (number, dimensions, position, etc.) and/or rules constraints like minimum dimension of an inspection window (product design constraints), allows to inference geometrical feature of a part.

Technological concepts and rules, for transforming design features in manufacturing features, essentially pertain mechanical and electrical engineering domains or generally speaking to physics. It is possible to formalize this knowledge as rules, to exploit concepts contained in IEC resource-models (material, equipment and personnel), as well as process segment- and product definition-models.

Resources constraints for managing process plan optimization are depending of single production site. These rules have a higher priority than manufacturing rules, since acts as constraints.

Rules and constraints based on experts knowledge, e.g. minimum material covering for a punching tool hit (technological knowledge) - machine $X$ does not allow manufacturing more than one negative bend (local resources constraints), allow to generate acceptable process planning.

Finally product design constraints are related to functional and/or regulated parts features or good practises. These constraints can be defined also for limiting sustainability suggestions.

\subsection{Other parts of the $K B S$}

In this section we design a KBS for exploiting the KB designed in the previous sections. The system has to help designers and production planners in automating their tasks and supporting them with sustainability suggestions.

We propose to build a KBS capable to easily adapt to different environments (production sites). We achieve a higher flexibility by formalizing, in the core system, only product manufacturing knowledge that is common for each production site and identifying site specific knowledge required by the system at site installation. Moreover we are preserving the same effectiveness as the installed system has all knowledge required for performing specific tasks.
In Fig. 7, a model using the BPMN notation (White et al., 2008) represents the typical system usage scenario. The system inputs are product specification whereas outputs are parts programs files. $\mathrm{KB}$ is connected to enterprise databases (DBs), which contain product-related information.

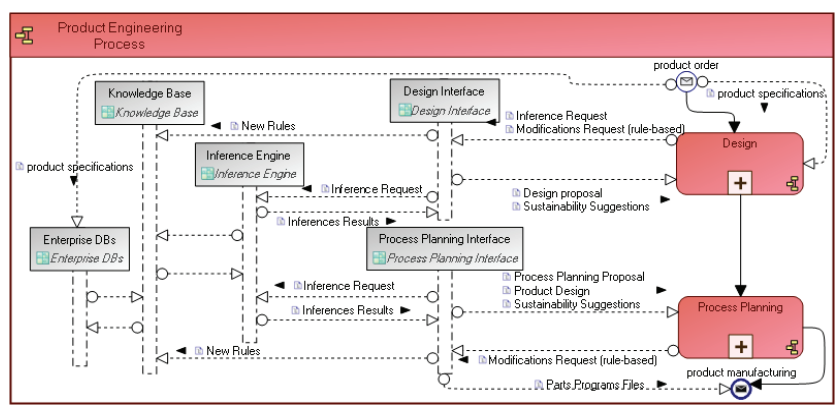

Fig. 7 - Key reference system usage scenario model.

Interfaces at design and process planning levels have three main functions:

- to allow experts interacting with $\mathrm{KB}$, through an $\mathrm{OWL}$ inference engine, and consequently with information in DBs;

- to acquire knowledge which allows expert enriching $\mathrm{KB}$; a part of this knowledge (e.g. machine code semantics) is required to system functioning; so interfaces are able to ask for information needed;

- to elaborate inferences results based on concepts and rules in the $\mathrm{KB}$; interfaces aim is to transform results based on technological and sustainable manufacturing knowledge in drawings and code; the output is consequently specific to each production site.

Moreover, interfaces allow interacting with outputs modifying inferences results. In fact designer can refuse proposals, editing manually CAD files or adding rules using the knowledge acquisition module included in the interface. In both cases, the system will present other design proposals until designers accept the solution. When a design proposal is inferred, at the interface, designers visualize sustainability suggestions, related to resources to use or process to perform, for manufacturing the product parts. Various more sustainable solutions are proposed, including information about reasons of suggestions and effects of the acceptance.

At process planning stage, the inferred process plan is received after designers accept system solution. At the interface, engineers can visualize design and process planning results. Visualizing design outputs allows process planners a better understanding of system results and therefore the possibility to propose, when required, appropriate modifications. Also in this interface, experts can introduce knowledge in the system using the knowledge acquisition module. Rules can affect design stage; in this case a newer designers' acceptance is required. When a process plan is inferred, related sustainability suggestions are proposed at the interface. At 
this interface, there are the same functionalities of designer one. Moreover, when an acceptance affects parts design, system sends at designer interface a notification for requiring a modifications acceptance.

When designer and process planners accept system solutions, process planning interface generates parts machine files. During all these stages, system use DBs information and finally updates contained information according final design and process planning solutions.

\section{CONCLUSIONS AND PERSPECTIVES}

In this paper we extend the ontology ONTO-PDM to make it able to perform suggestions based on manufacturing sustainability knowledge. We instantiate the model with a real case and finally we propose the design of a KBS that exploit this knowledge for supporting design and process planning.

The system is composed by a core that allows obtaining flexibility. The ideal application of this system is a multisite company scenario for which Trane is an example. In this type of organization, the same product can be manufactured in different factories around the world, with different resources, business rules and therefore different process planning results. Modifications (not at a functional level) on components design can be performed taking resources (machine, tools, etc.) into account. Therefore, in this scenario, it is required to have a deep knowledge about production sites peculiarities for introducing sustainability knowledge in manufacturing.

The next step is to implement the whole system and to test it in this scenario.

\section{REFERENCES}

Brilhante, V., Ferreira, A., Marinho, J. and Pereira, JS (2006), Information Integration Through Ontology and Metadata for Sustainability Analysis, , in: Proc. of the 3rd biennial meeting of the International Environmental Modelling and Software Society.

Ceccaroni, L., Cortés, U. and Sanchez-Marre, M. (2004), OntoWEDSS: Augmenting Environmental Decisionsupport Systems with Ontologies. Environmental Modelling \& Software, 19(9): 785-797.

Darai, D.S., Singh, S. and Biswas, S. (2010), Knowledge Engineering-an Overview. International Journal of Computer Science and Information Technologies, 1(4): 230-234.

Dori, D. and Shpitalni, M. (2005), Mapping Knowledge About Product Lifecycle Engineering for Ontology Construction via Object-process Methodology. CIRP Annals-Manufacturing Technology, 54(1): 117-122.

Freitas, F., Stuckenschmidt, H. and Noy, N.F. (2005), Ontology Issues and Applications. Journal of the Brazilian Computer Society, 11(2): 5-16.

Garrido, J. and Requena, I. (2011), Proposal of Ontology for Environmental Impact Assessment: An Application with Knowledge Mobilization. Expert Systems with Applications, 38(3): 2462-2472.
Gruber, T.R. (1993), A Translation Approach to Portable Ontology Specifications. Knowledge acquisition, 5(2): 199-220.

Harshit Kumar, H.K. and Pil Seong Park, P.S.P. (2010), Know-Ont: A Knowledge Ontology for an Enterprise in an Industrial Domain. International Journal of Database Theory and Application, 3(1): 23-32.

Horrocks, I., Patel-Schneider, P.F., Boley, H., Tabet, S., Grosof, B. and Dean, M. (2004), SWRL: A Semantic Web Rule Language Combining OWL and RuleML. W3C Member submission, 21: 79.

Iwata, K. (1988), Application of Expert Systems to Manufacturing in Japan. The International Journal of Advanced Manufacturing Technology, 3(3): 23-37.

Kibira, D., Jain, S. and Mclean, C. (2009), A System Dynamics Modeling Framework for Sustainable Manufacturing, , in: Proceedings of the 27th Annual System Dynamics Society Conference.

Lemaignan, S., Siadat, A., Dantan, J.Y. and Semenenko, A. (2006), MASON: A Proposal for an Ontology of Manufacturing Domain, 195-200, in: Distributed Intelligent Systems: Collective Intelligence and Its Applications, 2006. DIS 2006.

McGuinness, D.L. and Van Harmelen, F. (2004), OWL Web Ontology Language Overview. W3C recommendation, 10: 2004-03.

Panetto, H., Dassisti, M. and Tursi, A. (2012), ONTOPDM: Product-driven ONTOlogy for Product Data Management Interoperability Within Manufacturing Process Environment. Advanced Engineering Informatics.

Pham, DT and Pham, P.T.N. (1988), Expert Systems in Mechanical and Manufacturing Engineering. The International Journal of Advanced Manufacturing Technology, 3(3): 3-21.

Reyes-Cordoba, A P, Sharratt, P N and ArizmendiSanchez, J A (2008), Contribution of Knowledge Management for the Implementation of Waste Minimisation Measures into Process Industries. Process Safety and Environmental Protection, 86(5): 375-388.

Staab, S. (2009), Handbook on Ontologies. Springer Verlag.

Tursi, A., Panetto, H., Morel, G. and Dassisti, M. (2009), Ontological Approach for Products-centric Information System Interoperability in Networked Manufacturing Enterprises. Annual Reviews in Control, 33(2): 238-245.

U.S. Department of Commerce (2009), available at http://www.trade.gov/competitiveness/sustainableman ufacturing/

Wang, K. and Tong, S. (2008), An Ontology of Manufacturing Knowledge for Design Decision Support, 1-5, in: Wireless Communications, Networking and Mobile Computing, 2008. WiCOM'08.

White, S.A. and Miers, D. (2008), BPMN Modeling and Reference Guide. Future Strategies Inc. 